\title{
Editorial on quality standards in upper gastrointestinal endoscopy: a position statement of the British Society of Gastroenterology (BSG) and Association of Upper Gastrointestinal Surgeons of Great Britain and Ireland (AUGIS)
}

\author{
Philip Wai Yan Chiu \\ Division of Upper GI and Metabolic Surgery, Department of Surgery, Institute of Digestive Disease, The Chinese University of Hong Kong, Hong \\ Kong, China \\ Correspondence to: Professor Philip WY Chiu, MD, FRCSEd. 4th floor, Department of Surgery, Prince of Wales Hospital, 30-32 Ngan Shing Street, \\ Shatin, N.T., Hong Kong, China. Email: philipchiu@surgery.cuhk.edu.hk. \\ Provenance: This is a Guest Editorial commissioned by Editor-in-Chief Jia-Fu Ji (Peking University School of Oncology \& Beijing Cancer Hospital, \\ Department of Gastrointestinal Surgery, Beijing, China). \\ Comment on: Beg S, Ragunath K, Wyman A, et al. Quality standards in upper gastrointestinal endoscopy: a position statement of the British Society \\ of Gastroenterology (BSG) and Association of Upper Gastrointestinal Surgeons of Great Britain and Ireland (AUGIS). Gut 2017;66:1886-99.
}

Received: 05 December 2017; Accepted: 08 December 2017; Published: 28 February 2018.

doi: $10.21037 /$ tgh.2018.01.05

View this article at: http://dx.doi.org/10.21037/tgh.2018.01.05

Upper gastrointestinal (GI) endoscopy was generally considered as a simple investigation which does not require much skills to learn. Usually, it is considered as one of the first endoscopic procedures to be learned by trainees. Conventional diagnostic upper GI endoscopy is performed with white light endoscopy. However, with the recent advances in imaging technologies, image enhance endoscopy (IEE) has been increasingly applied especially during surveillance upper GI endoscopy. A systematic review of 22 studies showed that the missed rate of gastric cancer during upper endoscopy amounts to $9.4 \%$ (1). Missed cancers are usually located in gastric body both from Eastern and Western studies. Another systematic review on 24 studies focused on missed esophageal adenocarcinoma after diagnosis of Barrett's esophagus, and the results showed $25 \%$ of adenocarcinoma diagnosed had received an upper GI endoscopy within 1 year (2).

There is an unmet demand for better quality and key performance indicators during upper GI endoscopy. Beg et al. reported the position statement of the British Society of Gastroenterology (BSG) and Association of Upper Gastrointestinal Surgeons of Great Britain and Ireland (AUGIS) on quality standards for upper GI endoscopy (3). The standard of practice for diagnostic upper GI endoscopy is listed within the position statement, which aimed to establish the standards to allow quality assurance for upper GI endoscopy. A total of 38 statements were included with voting from panel members on the strength of recommendation, as well as the grade of evidence. These statements were developed according to the patient management pathway, including pre-procedure, procedure, disease specific and post-procedure management issues.

Especially important in this statement are the recommendations related to clinical practice of upper GI endoscopy to enhance detection of early upper gastrointestinal neoplasia. These included photos documentation at relevant anatomical landmarks and any detected lesions, adequate mucosal visualization, as well as inspection time during a diagnostic oesophagogastroduodenoscopy (OGD) to be recorded for surveillance procedures. These are important steps to ensure that those areas which are easily missed during diagnostic endoscopy will be inspected. The quality will be assured even when the experience in performing upper GI endoscopy and diagnosis of early upper GI neoplasia varies between trainees and specialists. Yao et al. proposed the performance of systematic screening of stomach (SSS) protocol where 22 pictures should be taken during upper 
endoscopy in stomach to avoid blind spots (4). Teh et al. in a single-center retrospective trial showed that endoscopists with mean EGD time longer than 7-minute were twice as likely to detect high-risk lesions (OR 2.50; 95\% CI: 1.52$4.12)$ and three-fold as likely to detect neoplastic lesions (OR 3.42; 95\% CI: 1.25-10.38) (5). A standard protocol for upper GI endoscopy is essential to ensure good quality, and not missing all the precancerous and early cancers in esophagus and stomach (6). Typically, these early neoplasias demonstrated subtle changes in the mucosa, represented by changes in microvascular and microstructural patterns in stomach and changes in intrapapillary capillary loops (IPCL) in squamous mucosal of esophagus.

A significant number of statements focused on need to take biopsies for various lesions detected during upper endoscopy (3). The diagnostic process of upper GI neoplasia includes detection upon ordinary white light endoscopy or IEE, followed by characterization with recognition of microstructural and microvascular features observed under magnification (7-9). With regards to the disease specific quality standards, the guidelines proposed adoption of numerous classification to describe various disease pathologies during endoscopy. The Prague classification was recommended to describe length and circumferential extend of Barrett's segment, which has high interobserver agreement (10). Paris classification is recommended upon detection of a suspicious neoplastic lesion of the gastrointestinal tract (11). Paris classification served as a universal description for macroscopic appearance of the lesion, thus assisting in standardization of understanding the morphological appearance and enhance communication. Taking biopsies at suspicious lesions will improve the process of endoscopic diagnosis even for endoscopists without knowledge on characterization under magnifying endoscopy. In one of the statements, the authors recommended for a malignant looking lesion, a minimum of six biopsies should be taken. There was 100\% agreement with strong recommendation, though grade of evidence was weak. The authors argued that minimum six biopsies allowed prompt establishment of the diagnosis without the need for repeated examination. Though this approach would be appropriate for an obviously malignant tumor with invasion to muscularis propria or beyond, taking six biopsies from an early gastrointestinal cancer would induce significant fibrosis in submucosal plane, leading to a more difficult endoscopic resection in future (12).

The guidelines also proposed to set up an audit on the rate of failure to diagnose cancer at endoscopy up to 3 years before an oesophago-gastric cancer was diagnosed. Though there is minimal evidence in literature, the statement is strongly supported by the panel. Retrospective studies have shown that the rate of post-OGD Upper GI cancer (POUGIC) ranges between $4.6 \%$ and $14.4 \%(13,14)$. The panel recommends that individual endoscopy unit should audit performance data to ensure that POUGIC rates should not exceed $10 \%$. Though a wide range of parameters existed in missing an upper GI cancer, an audit will contribute to improvement through the process of identifying root cause of missing the upper GI cancer.

In summary, this positional statement of the BSG and AUGIS serves as an important guide to ensure a good quality of upper gastrointestinal endoscopy, which fills the important gap between the high technical success of upper GI endoscopy and the relatively low diagnosis of early upper GI cancers in most countries.

\section{Acknowledgements}

None.

\section{Footnote}

Conflicts of Interest: The author has no conflicts of interest to declare.

\section{References}

1. Pimenta-Melo AR, Monteiro-Soares M, Libânio D, et al. Missing rate for gastric cancer during upper gastrointestinal endoscopy: a systematic review and metaanalysis. Eur J Gastroenterol Hepatol 2016;28:1041-9.

2. Visrodia K, Singh S, Krishnamoorthi R, et al. Magnitude of Missed Esophageal Adenocarcinoma After Barrett's Esophagus Diagnosis: A Systematic Review and Metaanalysis. Gastroenterology 2016;150:599-607.

3. Beg S, Ragunath K, Wyman A, et al. Quality standards in upper gastrointestinal endoscopy: a position statement of the British Society of Gastroenterology (BSG) and Association of Upper Gastrointestinal Surgeons of Great Britain and Ireland (AUGIS). Gut 2017;66:1886-99.

4. Yao K. The endoscopic diagnosis of early gastric cancer. Ann Gastroenterol 2013;26:11-22.

5. Teh JL, Tan JR, Lau LJ, et al. Longer examination time improves detection of gastric cancer during diagnostic upper gastrointestinal endoscopy. Clin Gastroenterol Hepatol 2015;13:480-487.e2. 
6. Rey JF, Lambert R; ESGE Quality Assurance Committee. ESGE recommendations for quality control in gastrointestinal endoscopy: guidelines for image documentation in upper and lower GI endoscopy. Endoscopy 2001;33:901-3.

7. Muto M, Yao K, Kaise M, et al. Magnifying endoscopy simple diagnostic algorithm for early gastric cancer (MESDA-G). Dig Endosc 2016;28:379-93.

8. Nagami Y, Tominaga K, Machida H, et al. Usefulness of non-magnifying narrow-band imaging in screening of early esophageal squamous cell carcinoma: a prospective comparative study using propensity score matching. Am J Gastroenterol 2014;109:845-54.

9. Qumseya BJ, Wang H, Badie N, et al. Advanced imaging technologies increase detection of dysplasia and neoplasia in patients with Barrett's esophagus: a meta-analysis and systematic review. Clin Gastroenterol Hepatol 2013;11:1562-70.e1-2.

doi: 10.21037/tgh.2018.01.05

Cite this article as: Chiu PW. Editorial on quality standards in upper gastrointestinal endoscopy: a position statement of the British Society of Gastroenterology (BSG) and Association of Upper Gastrointestinal Surgeons of Great Britain and Ireland (AUGIS). Transl Gastroenterol Hepatol 2018;3:13.
10. Alvarez Herrero L, Curvers WL, van Vilsteren FG, et al. Validation of the Prague C \&M classification of Barrett's esophagus in clinical practice. Endoscopy 2013;45:876-82.

11. The Paris endoscopic classification of superficial neoplastic lesions: esophagus, stomach, and colon: November 30 to December 1, 2002. Gastrointest Endosc 2003;58:S3-43

12. Higashimaya M, Oka S, Tanaka S, et al. Outcome of endoscopic submucosal dissection for gastric neoplasm in relationship to endoscopic classification of submucosal fibrosis. Gastric Cancer 2013;16:404-10.

13. Yalamarthi S, Witherspoon P, McCole D, et al. Missed diagnoses in patients with upper gastrointestinal cancers. Endoscopy 2004;36:874-9.

14. Raftopoulos SC, Segarajasingam DS, Burke V, et al. A cohort study of missed and new cancers after esophagogastroduodenoscopy. Am J Gastroenterol 2010;105:1292-7. 